\title{
AZT Treatment Increases mtDNA Mutations in HepG2 and CCD-1112Sk Cells
}

\author{
Adam E. Osborne*, John E. Rice, J Aquiles Sanchez and Lawrence J. Wangh \\ Biology, Brandeis University, Waltham, USA
}

\begin{abstract}
Mitochondrial dysfunction is linked to disease, but it remains unclear whether accumulation of random mutations in the mitochondrial genome is the cause of dysfunction. Using digital or near-digital LATE-PCR with Lights-On/LightsOff probes we have measured the mutational load in mitochondrial genomes. Exposure of HepG2 and CCD-1112Sk cells to AZT for thirty days caused a significant increase in mutations in the three mitochondrial loci examined. These results demonstrate the utility of our method for analysis of mutational load without sequencing and reinforce the fact that mitochondrial DNA damage due to drugs, aging, and disease should be studied in detail.
\end{abstract}

Keywords: Mitochondria; LATE-PCR; Digital PCR; AZT; NRTI; HIV/AIDS; Heteroplasmy

\section{Introduction}

Every eukaryotic cell has hundreds or even thousands of mitochondria which regulate cellular energetics and metabolism. Mitochondrial dysfunction increases over time and is observed as an accumulation in reactive oxygen species (ROS), as decreases in synthesis of electron transport chain proteins and ATP production, and/or as changes in mitochondrial size, shape, and membrane potential. This dysfunction has been linked to aging [1-3] and a host of age-related diseases including Parkinson's [4], Alzheimer's [5,6], diabetes [7], and others $[8,9]$. Recent studies have also shown that patients on long term HIV/AIDS therapy exhibit many mitochondria related diseases including, diabetes, Parkinson's, and Alzheimer's [10,11]. These reports are consistent with the evidence that mitochondrial dysfunction is the side effect of drug treatment [12-14].

A possible cause of mitochondrial dysfunction is damage to the many copies of mitochondrial DNA (mtDNA). One form of this damage is the gradual accumulation of mtDNA mutations [1]. These mutations may adversely affect mitochondrial function, or may cause an increased production of reactive oxygen species (ROS), initiating a feedback loop of mutations and further ROS generation. Alternatively, mitochondria may also become dysfunctional due to the depletion of genomes. In fact, certain antiretroviral drugs, such as nucleoside reverse-transcriptase inhibitors (NRTIs), used to treat HIV/AIDS, inhibit DNA replication [15], resulting in mtDNA depletion [16,17]. The NRTI 3'-Azido-3'-deoxythymidine (AZT also known as ZDV) in contrast, does not appear to cause mtDNA depletion, but nevertheless causes mitochondrial dysfunction [18-20].

AZT may cause dysfunction by damaging mtDNA [21]. This damage may come in the form of low frequency random mutations, known as mutational load, which may build up gradually until mitochondria become dysfunctional and clinical symptoms arise. Thus, mutational load rather than depletion of mtDNA could account for the negative effects of AZT treatment. According to this hypothesis no single mutation reaches a threshold to cause dysfunction, but all mutations collectively cause dysfunction, which in turn leads to disease. Alternatively mutational load could lower the threshold needed for point mutations to cause dysfunction and thus lead to disease. In either case, mutational load does not reach the threshold necessary for apoptosis due to its low frequency and random nature [22].
Most methods currently used to study mutational load are based on conventional PCR amplification followed by Sanger sequencing. These approaches are inadequate because they start with populations of molecules and therefore can only detect high frequency changes in mtDNA. Mutational load by its very nature is not high frequency. In fact, failure to detect mutational load may account for studies that show either no consensus in mutations as they relate to disease onset or mitochondrial dysfunction [23,24]. Two studies have addressed the question of mutational load with the proper detection resolution. Martin et al. [25] examined peripheral-blood samples taken prior to and after NRTI therapy and observed drug-induced sequence changes without evidence for positive selection of pre-existing somatic mutations. In contrast, Payne et al. [26] examined muscle fibres defective for mitochondrial function in naïve and NRTI-treated patients and concluded that drug treatment led to clonal expansion of pre-existing mutations rather than increased mutagenesis.

This paper investigates whether peak plasma levels of AZT, $7 \mu \mathrm{M}$ [27], damage mitochondrial genomes by increasing mutational load in either of two different cell lines. This was accomplished by analysing mutations in mtDNA at the digital or near-digital (1-5 copies) level. Analysis of single molecules not only allows the cataloguing of mutations, but also establishes the context in which those mutations occur. Single molecule amplification via LATE-PCR (an advanced form of non-symmetric PCR), followed by dideoxy sequencing has been shown to reliably detect mutations in mitochondrial DNA [28]. The drawback of this method is that hundreds of individual mitochondrial genomes have to be sequenced to properly measure mutational load. The present paper describes the use of LATE-PCR together with a novel probe design called Lights-On/Lights-Off probes [29] to simultaneously screen multiple loci in individual mitochondrial genomes for sequence changes. These probes generate fluorescent signatures that are unique

*Corresponding author: Adam E Osborne, Biology, Brandeis University, Waltham, MA, 02454, USA, Tel: 1-781-736-3111; Fax: 1-781-736-3107; E-mail: aosborne@brandeis.edu

Received August 07, 2013; Accepted September 29, 2013; Published October 11,2013

Citation: Osborne AE, Rice JE, Sanchez AJ, Wangh LJ (2013) AZT Treatment Increases mtDNA Mutations in HepG2 and CCD-1112Sk Cells. J AIDS Clin Res 4: 250. doi: $10.4172 / 2155-6113.1000250$

Copyright: (C) 2013 Osborne AE, et al. This is an open-access article distributed under the terms of the Creative Commons Attribution License, which permits unrestricted use, distribution, and reproduction in any medium, provided the original author and source are credited. 
to each amplified sequence. Any change from a reference fluorescent signature indicates the presence of a mutation. This approach greatly diminishes the need for sequencing because only signatures that differ from the reference are screened and, if desired, sequenced to identify the exact nucleotide change.

In this study HepG2 and CCD-1112Sk cells were treated with 7 $\mu \mathrm{M}$ AZT in culture for thirty days. Mitochondrial genomes were then interrogated at the digital or near-digital level using a triplex LATEPCR Lights-On/Lights-Off assay. Treatment did not deplete the mtDNA over that period of time, but caused a statistically significant increase in the frequency of random mutations as compared to untreated cells. This increase was observed in all three mtDNA loci examined: hyper variable region 2 (HV2), cytochrome c oxidase subunit 2 (CO2), and $\mathrm{NADH}$ dehydrogenase, subunit 1 (ND1). The presence and identity of the mutations were validated by sequencing in a subset of cases. These results demonstrate that AZT introduces low frequency sequence changes in mtDNA and illustrates the value of LATE-PCR with LightsOn/Lights-Off probes for analysis of mitochondria mutational load.

\section{Materials and Methods}

\section{Culture conditions and AZT treatments}

HepG2 liver carcinoma cells and CCD-1112Sk skin fibroblast cells were obtained from ATCC (Manassas, VA). HepG2 liver carcinoma cells are a good model for studying the effects of drugs such as AZT on mtDNA [20]. CCD-1112Sk skin fibroblasts are primary cells that served as a control for the HepG2 cancer cells which do not need mitochondria to grow [30]. Both cell lines were cultured for thirty days in the presence or absence of $7 \mu \mathrm{M}$ AZT (Sigma, St. Louis, MO). Cells were grown in Eagle's Minimum Essential Medium (HepG2 cells) or in Iscove's Modified Dulbecco's Medium (CCD-1112Sk cells) obtained from ATCC supplemented with $10 \%$ fetal bovine serum (BioWest, Kansas City, MO), 50 units/mL Penicillin G, 50 units/mL Streptomycin, $0.25 \mu \mathrm{g}$ Amphotericin B (HyClone Antibiotic/Antimycotic Solution $100 \mathrm{X}$ ) and were incubated at $37^{\circ} \mathrm{C}$ and $5 \% \mathrm{CO}_{2}$. The media was changed every other day. Cell lines were grown to confluency, and then trypsinized with $0.25 \%$ trypsin (Sigma, St. Louis, MO). After 30 days culture cells were collected and frozen in complete media with $5 \% \mathrm{v} / \mathrm{v}$ DMSO in liquid nitrogen. Three biological replicates were assessed, each with three technical replicates for each condition.

\section{Preparation of mitochondrial DNA}

Mitochondrial DNA was isolated as previously described [28]. Both treated and untreated samples were processed in parallel to avoid introducing any bias. Briefly, one microliter of cultured cells (roughly 1000 cells) was lysed in $14 \mu \mathrm{l}$ of Quantilyse [31]. Before PCR amplification mitochondrial genomes were cut once with BamH1 (Promega, Madison, WI), 37C for 1 hour followed by $74^{\circ} \mathrm{C}$ for 15 minutes. The digestion was carried out in a $20 \mu \mathrm{l}$ volume consisting of $1 \mathrm{X}$ reaction enzyme buffer, $0.1 \mathrm{mg} / \mathrm{ml} \mathrm{BSA}, 10 \mu \mathrm{lmtDNA}$ from the above lysate, and 10 units of BamH1. Linearized samples were stored at $-20^{\circ} \mathrm{C}$.

Digital and near-digital LATE-PCR using Lights-On/LightsOff probes for mutation detection

Assay primers and probes: Lysed DNA samples were first diluted to the digital or near-digital level (1-5 copies) as described in Osborne, 2009. To avoid contamination all PCR preparation was conducted in an amplicon-free hood in a clean room, all targets were kept separate from PCR reagents at all times, and PCR machines were kept in a separate room from the laboratory space as far away from the clean room as possible. PCR amplicons and products were never handled in the same laboratory space.

The triplex LATE-PCR amplification used three pairs of primers that co-amplified the mitochondrial hyper variable 2 (HV2) region of the D-Loop, the cytochrome c oxidase subunit $2\left(\mathrm{CO}_{2}\right)$ gene, the NADH dehydrogenase, subunit 1 (ND1) gene, and all the primers were checked using BLAST and an in-silico PCR program [32] to establish that they would not amplify nuclear pseudogenes. The underlined bases in the limiting primers and probes were deliberately mis-matched to the target sequence.

HV2 Limiting Primer: 5’ AAAGCGGTGTGTGTGTGCTGGGTAGGAT

HV2 Excess Primer: 5' ACTTCAGGGTCATAAAGCCTAAATAGC

CO2 Limiting Primer: 5’ AATAGAGGGGGTAGAGGGGGTGCTATAGGGT

CO2 Excess Primer: 5’ TCCTTATCTGCTTCCTAGTCCTGTATGC

ND1 Limiting Primer: 5' AACATAAGAACAGGGAGGTTAGAAGTAGGGTCTTGGT

\section{ND1 Excess Primer: 5' CGCCCCGACCTTAGCTCT}

Each pair of primers generated a specific product (HV2, 588 base pairs; $\mathrm{CO}_{2}, 586$ base pairs; and ND1, 604 base pairs long). Each of the three corresponding single-stranded amplicons was interrogated with its own set of Lights-On/Lights-Off probes labelled in its own colour and spanning a portion of its total length: HV2, 250 bases; CO2, 300 bases; ND1, 250 bases (Supplementary Table 1).

\section{Reagents and reaction conditions}

A common master mix was used for all experiments. The master mix consisted of 1X PCR buffer (Invitrogen, Carlsbad, CA), $3 \mathrm{mM}$ $\mathrm{MgCl}_{2}, 250 \mathrm{nM}$ dNTPs, $50 \mathrm{nM}$ Limiting Primer, $1000 \mathrm{nM}$ Excess Primer (HV2), 100 nM Limiting Primer, 1000 nM Excess Primer (CO2), $50 \mathrm{nM}$ Limiting Primer, 1500 nM Excess Primer (ND1), 2.5 units of Platinum Taq DNA Polymerase (Invitrogen, Carlsbad, CA), $50 \mathrm{nM}$ of the on probes, and $150 \mathrm{nM}$ of the off probes in $25 \mu \mathrm{l}$ reaction volumes. The PCR protocol was $95^{\circ} \mathrm{C}$ for 3 minutes, followed by $95^{\circ} \mathrm{C}$ for 5 seconds, $65^{\circ} \mathrm{C}$ for 45 seconds, and $72^{\circ} \mathrm{C}$ for 90 seconds for 75 cycles. This was followed by two holding cycles, the first at $75^{\circ} \mathrm{C}$ for 10 minutes, and the second at $25^{\circ} \mathrm{C}$ for ten minutes. Probe signals were acquired during a melt from $25^{\circ} \mathrm{C}$ to $80^{\circ} \mathrm{C}$ in increments of $1^{\circ} \mathrm{C}$ every 45 seconds.

\section{Analysis of fluorescent signatures and their changes}

Fluorescent signatures characteristic of each amplicon were generated by plotting the first derivative of probe signals as a function of temperature. Changes in the signature, i.e., shifts to lower or higher temperature or appearance or disappearance of peaks and valleys, relative to a reference signature were indicative of mutations. To obtain reference signatures, 12-14 bulk reactions containing over 1000-copies of mtDNA each were amplified and the resulting fluorescent signatures were averaged together (Supplementary Figure 1).

In any PCR experiment there are multiple sources of noise or error that can make interpretation of a signature shift difficult. Using one PCR master mix eliminates pipetting error and day-to-day variance of reagents. However, there is still well-to-well variation that can be generated by the PCR machine itself. In order to minimize this machine noise it was necessary to generate not only a reference signature as 
described above, but also to calculate the $95 \%$ confidence interval of each reference signature due to machine noise beyond which mutations were scored (Supplementary Figure 1). These confidence intervals were defined as three standard deviations of the largest temperature variation among all reference replicates at the highest or lowest fluorescent values for each signature. Shifted signatures from test samples that had more than two temperature points outside these confidence intervals were considered a true shift due to mutation rather than noise. These objective criteria for scoring mutations eliminate subjective bias during mutation assessment. The statistical significance of true shifts was calculated using a chi-square test.

\section{Validation of mutant fluorescent signatures}

A number of samples with signature shifts were randomly selected for validation by Sanger sequencing. Samples were prepared by the Dilute-'N'-Go protocol described previously $[28,33]$. If necessary, the opposite strand was also sequenced to confirm any mutations using the method of Jia et al. [34]. Sequencing was performed at Genewiz, Inc. (New Jersey).

\section{Measurement of mtDNA copy number}

Assay primers and probes: The effect of AZT treatment on mtDNA copy number was assessed by measuring the ratio of mitochondrial to nuclear DNA (nDNA). A mitochondrial gene and its corresponding nuclear pseudogene were co-amplified with LATE-PCR using limiting primers specific for each target and a common excess primer to achieve the same amplification efficiency. The amplification products were detected with two amplicon specific probes whose signal ratio at the threshold cycle were used to establish the relative amounts of mitochondrial and nuclear DNA. The underlined bases in the limiting primers and probes sequences below are mis-matched to the target sequence.

mtDNA Limiting Primer: 5’ AACATAGGGTCTTCTCGTCTTGCTGTGTTATGC

nDNA Limiting Primer: 5’ AAGTCGACTCCGTGGCTCTCTCAGATCATC

Common Excess Primer: 5' TTTTGCCCCGCCTGTTTACC

mtDNA Probe: 5' Cal Red 590-GTATACTGGTGATGCTAGAGAC-BHQ2

nDNA Probe: 5' Quasar 670-TTCCGTGGACGTTAGACACATTTCAA-BHQ2

Reagents and reaction conditions: LATE-PCR amplifications were carried out in a $25 \mu \mathrm{l}$ volume consisting of $1 \mathrm{X}$ PCR buffer (Invitrogen, Carlsbad, CA), $3 \mathrm{mM} \mathrm{MgCl}$, $250 \mathrm{nM}$ dNTPs, $50 \mathrm{nM}$ each Limiting Primer, $1000 \mathrm{nM}$ Excess Primer, $150 \mathrm{nM}$ of each probe, and 1.25 units of Platinum Taq DNA Polymerase (Invitrogen, Carlsbad, CA). All amplifications were carried out in the Stratagene MX3005P (Agilent Technologies, CA). The reaction mixture was initially heated to $95^{\circ} \mathrm{C}$ for 3 minutes, then 10 cycles of $95^{\circ} \mathrm{C}$ for 5 seconds, $65^{\circ} \mathrm{C}$ for 20 seconds, and $70^{\circ} \mathrm{C}$ for 45 seconds. This was followed by 50 cycles of $95^{\circ} \mathrm{C}$ for 5 seconds, $65^{\circ} \mathrm{C}$ for 20 seconds, $70^{\circ} \mathrm{C}$ for 45 seconds, and $50^{\circ} \mathrm{C}$ for 42

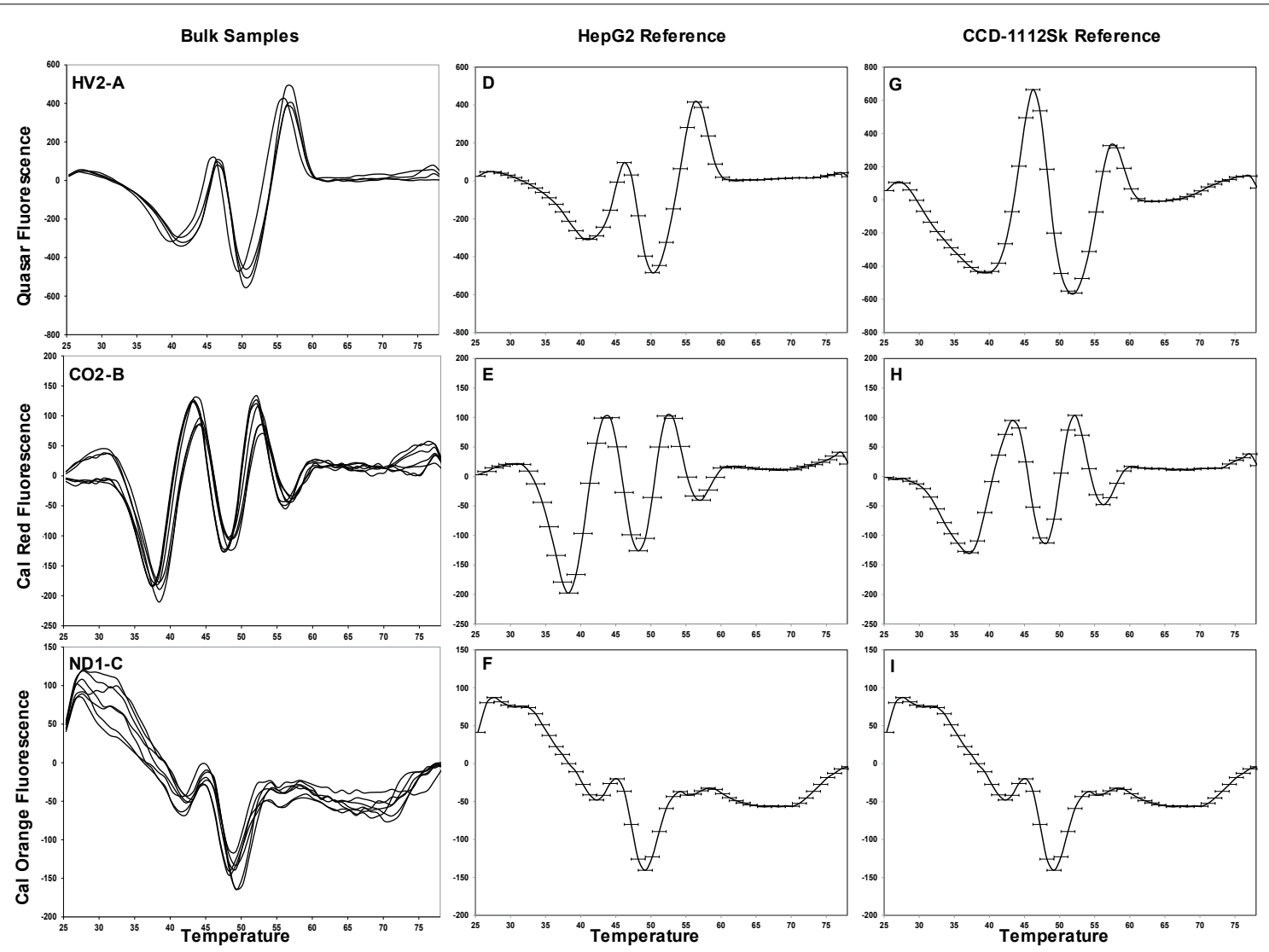

Figure 1: Reference fluorescent signatures for the HepG2 and CCD-1112Sk cell lines. Multiple bulk samples (approximately 1000-genomes each) analyzed and averaged $(A-C)$. Confidence intervals (three standard deviations) were then applied to the signature (D-F). Any signature shift that fell outside of this reference was considered a true shift. The same was done for the CCD-1112Sk amplicons (G-I). 
seconds. The amplification was read in real time at the $50^{\circ} \mathrm{C}$ step. The $\mathrm{C}_{\mathrm{t}}$ values collected during the amplification were used to determine the nDNA/mtDNA ratio.

\section{Results}

\section{Controls to rule out sample contamination}

Detection of mtDNA mutational load requires analysis of mitochondrial genomes at the digital or near-digital level (1-5 copies). Laboratory contamination is always a risk when working at such low copy numbers [28]. Control experiments showed that none of the sequence variants scored could be attributed to contamination from laboratory personnel or reagents (data not shown). Among those experiments one of 96 control reactions without mtDNA generated only a HV2 amplicon and two of 96 replicates generated only $\mathrm{CO}_{2}$ amplicons. No single replicate generated all three amplicons at the same time. Thus it is very unlikely that the shifts in the fluorescent signatures reported here were due to laboratory contamination.

\section{LATE-PCR Lights-On/Lights-Off analysis of mitochondrial mutational load}

The LATE-PCR mitochondrial triplex assay co-amplifies three mitochondrial loci (HV2, $\mathrm{CO}_{2}$, and $\mathrm{ND} 1$ ) as separate amplicons in a single tube. Mitochondrial DNA isolated from treated or untreated cells were first diluted to the digital or near-digital level and then amplified in the above triplex reaction. The resulting single-stranded DNA products were then examined for sequence variations using

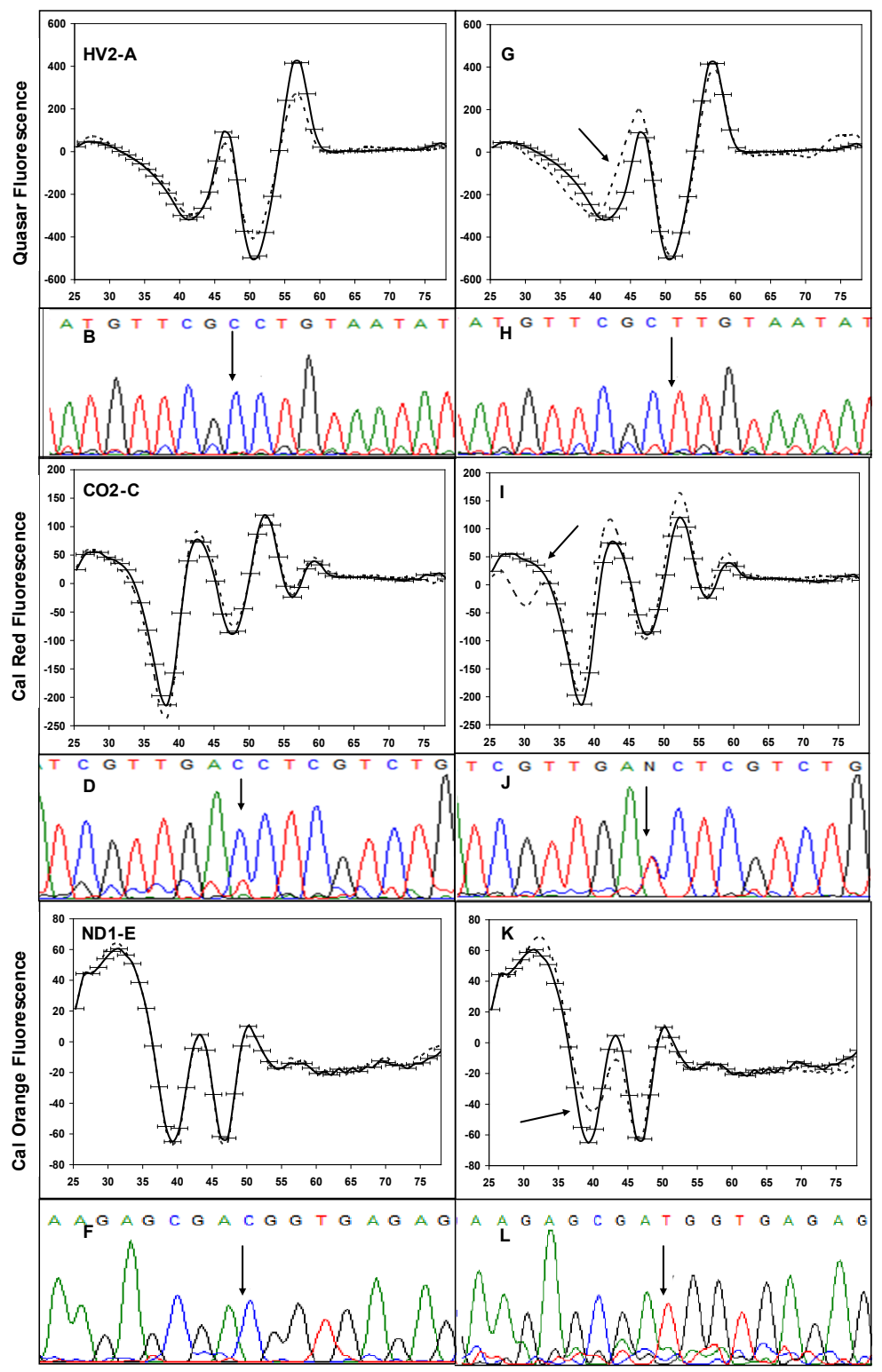

Figure 2: Every change in fluorescent signature equates to a change in the sequence of the amplicon. Representative single molecules of the three amplicons, HV2, $\mathrm{CO} 2$, and ND1 are shown. Panels A, E, and I show a single molecule that matches the reference. The sold lines indicate the reference signature, while the dotted lines indicate the single molecule. Panels $\mathrm{B}, \mathrm{F}$, and $\mathrm{J}$ show a single molecule that differs from the reference signature. The arrows in panels $\mathrm{B}$, $\mathrm{F}$, and $\mathrm{J}$ indicate where the single molecule signature differs from the reference signature. The arrows in panels $\mathrm{C}, \mathrm{G}$ and $\mathrm{K}$ show the nucleotide in the reference sequence that is changed in panels $\mathrm{D}, \mathrm{H}$, and L. 
three sets of Light-On/Lights-Off probes fluorescently labeled in different colors (Supplementary Table 1). Lights-On/Lights-Off probes generate fluorescent signatures that are characteristic of the amplified sequence. Any changes from a reference fluorescent signature indicate the presence of a mutation. The reference fluorescent signature for each amplicon in both cell types is comprised of the average fluorescent signature from 12-14 replicate samples, each containing more than 1,000 mtDNA molecules, along with the 95\% confidence interval that accounts for tube-to-tube variations in the thermal bloc (Figure 1, Supplementary Figure 1).

Figure 2, panels A-F, illustrates that the majority of fluorescent signatures in digital or near-digital samples are indistinguishable from the reference signatures. But Figure 2, panels G-L, illustrates that a minority of these samples fell outside of the confidence interval of the reference signature. Sanger sequencing of these cases confirmed that each of them contained specific sequence variations (Figure $2 b, d, f, h, j$, and 1 ). In no case did a digital or near-digital sample with a fluorescent signature shift not have a sequence change. These results validate that every time there is a signature shift there is a corresponding mutation or mutations.

\section{AZT treatment correlates with an increase in mtDNA mutations}

In order to determine whether AZT causes mtDNA mutational load HepG2 and CCD-1112Sk cells were treated with peak plasma levels of AZT for 30 days, and examined at the digital or near digital levels as described above. A total of 2,446 wells with 1- 5 copies of starting mtDNA (an estimated 12,240 amplicons total) were examined in three separate experiments.

All three gene targets in both HepG2 and CCD-1112Sk cells had a statistically significant increase in the number of fluorescent signature shifts in the AZT treated samples as compared to the non-treated samples (Figure 3 and 4 and Tables 1-3). Changes in the HV2 amplicon involved shifts to the left of a peak in the fluorescent signature (Figure $3 \mathrm{a}$ ), as well as the disappearance or appearance of a peak (Figure 3b and c). The fluorescent signature for the ND1 amplicons exhibited similar changes (Figure 3g-i). Many of the shifts seen in the $\mathrm{CO} 2$ fluorescent signature were not shifts to the left, but rather to the right (Figure 3d), as well as peaks that disappeared or broadened (Figure $3 \mathrm{e}$ and $\mathrm{f}$ ). In addition, the three amplicons of the CCD-1112Sk cells had a similar number and type of shifts as those observed in the HepG2 samples (Table 1). Selected samples with signature shifts were validated by sequencing (Table 3 ). This analysis revealed that some of the fluorescent signature shifts were the result of more than one mutation. In no instance did a change in a fluorescent signature occur without a change in target sequence.

Combining the results from each of the thirty day experiments yielded mutational frequencies that were higher for the AZT treated samples compared to non-treated samples (Table 2). Indeed, all AZT treated samples had higher rates of mutation. In the case of the HV2
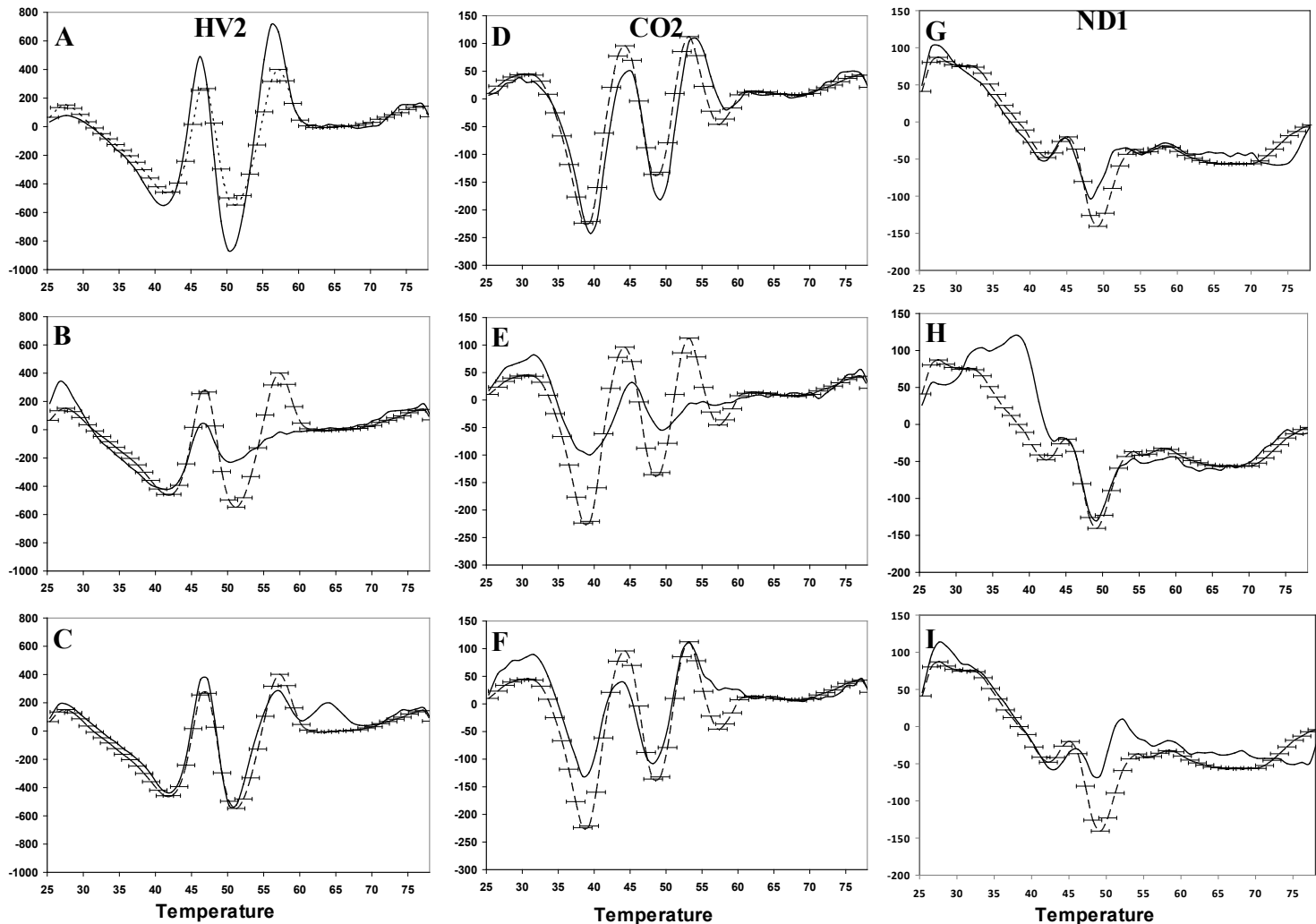

Figure 3: Mutations in the mitochondrial DNA target result in shifts in the fluorescent signature for amplification of HV2, ND1, and CO2. Representative shifts in the fluorescent signatures for the three amplicons in the triplex reaction. Panels A-C show shifts from the HV2 amplicon, D-F show shifts from the CO2 amplicon, and G-I show shifts from the ND1 amplicon. The dotted line is the reference signature the solid line is the experimental sample. The error bars are three standard deviations from the reference signature. 

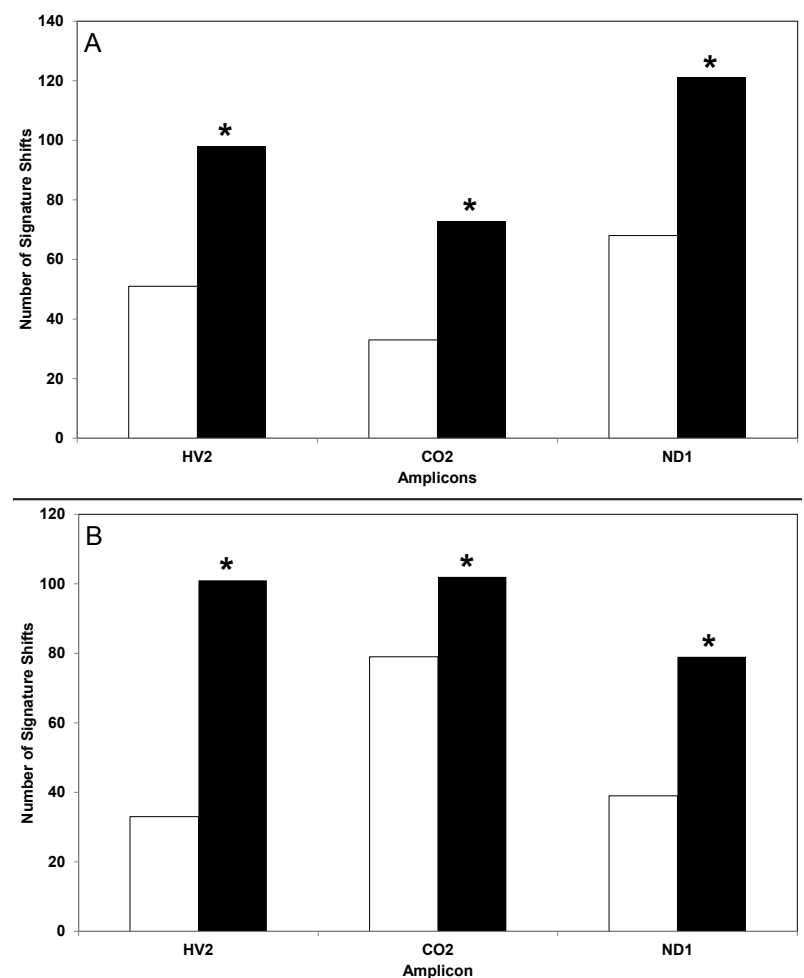

Figure 4: Thirty days of AZT treatment results in an increase in mutations in the mitochondrial genome. The number of shifts, and therefore mutations, by amplicon, observed for the HepG2 (A) and CCD-1112Sk (B) cell lines after thirty days of AZT treatment. The combined results for all replicates for each experiment are in black for the AZT treated samples, and are in white for the non-AZT treated samples. $A$ * represents those samples that have a p-value of 0.05 or less.

Table 1: Number of fluorescent signature shifts seen after thirty days $\left(T_{30}\right)$ with and without AZT treatment.

\begin{tabular}{|c|c|c|c|c|}
\hline & & $\mathrm{T}_{30}+\mathrm{AZT}$ & $\mathrm{T}_{30}-\mathrm{AZT}$ & p-value \\
\hline \multirow{3}{*}{ HepG2 } & $\mathrm{HV} 2$ & 98 & 51 & 0.00001 \\
\cline { 2 - 5 } & $\mathrm{CO} 2$ & 73 & 33 & 0.00002 \\
\cline { 2 - 5 } & $\mathrm{ND} 1$ & 116 & 68 & 0.00003 \\
\hline \multirow{3}{*}{ CCD-1112Sk } & $\mathrm{HV} 2$ & 101 & 33 & $1 \mathrm{e}-8$ \\
\cline { 2 - 5 } & $\mathrm{CO} 2$ & 102 & 79 & 0.05 \\
\cline { 2 - 5 } & $\mathrm{ND} 1$ & 79 & 39 & 0.0001 \\
\hline
\end{tabular}

The table shows the number of shifts for all experiments. For the three 30 day experiments 783/789 (treated/untreated) wells were analyzed for the HepG2 cells. For the CCD-1112Sk cells 781/798 wells were analyzed. A total of 2,440 wells, an estimated 12,240 mtDNA molecules, were analyzed. P-values were calculated by a chi-square test.

Table 2: The frequency of mutations seen in treated and non-treated cells

\begin{tabular}{|c|c|c|c|c|}
\hline & HepG2 AZT- & HepG2 AZT+ & CCD-1112Sk AZT- & CCD-1112Sk AZT+ \\
\hline HV2 & $2.2 \times 10^{-5}$ & $4.2 \times 10^{-5}$ & $1.4 \times 10^{-5}$ & $4.3 \times 10^{-5}$ \\
\hline CO2 & $1.4 \times 10^{-5}$ & $3.1 \times 10^{-5}$ & $3.3 \times 10^{-5}$ & $4.4 \times 10^{-5}$ \\
\hline ND1 & $2.9 \times 10^{-5}$ & $5.2 \times 10^{-5}$ & $1.6 \times 10^{-5}$ & $3.4 \times 10^{-5}$ \\
\hline
\end{tabular}

For both HepG2 and CCD-1112Sk cells, the frequency of mutations was higher for the AZT treated samples.

and ND1 targets the rate was twice as high for both cell types.

\section{AZT does not deplete mitochondrial DNA}

There are conflicting results in the literature as to whether AZT does [17] or does not [18] cause mitochondrial DNA depletion. It was therefore necessary to establish whether AZT treatment resulted in mtDNA depletion in the two cell lines used here. The ratio of nuclear DNA to mitochondrial DNA (see Material and Methods) before addition of AZT and after thirty days of treatment was unchanged (data not shown). Therefore, we conclude that $7 \mu \mathrm{M}$ of AZT does not cause mtDNA depletion in cultured HepG2 and CCD-1112Sk cells.

\section{Discussion}

Long-term treatment with NRTIs, which AZT, used by millions of individuals, is but one example, causes a series of pathologies associated with mitochondrial toxicity. To date, the mechanism of AZT-induced mitochondrial toxicity is still not clear. Indeed, the mutagenic effect of AZT and other NRTIs on mitochondrial DNA in vivo is controversial $[25,26]$. In part this is because these drugs are often prescribed as one component of highly active antiretroviral cocktails of drugs, which also may be increasing mutational load. In addition, HIV infection itself can damage mitochondria [35,36]. Thus, under normal conditions there are numerous compounding factors that likely increase mutational load over time in many cells and entire organs.

This report demonstrates that AZT treatment for thirty days causes

Table 3: Representative mutations found in randomly selected treated and untreated samples with signature shifts for all three amplicons.

\begin{tabular}{|c|c|c|}
\hline Treatment & Amplicon & Mutation \\
\hline \multirow[t]{3}{*}{ AZT Treated } & HV2 & $\begin{array}{l}11 A \\
24 A \\
64 T \\
80 A \\
128 C-382 A-396 C \\
181 A \\
198 G \\
295 A \\
320 A-345 A \\
369 A-382 C-388 A \\
369 A-388 A \\
382 A-396 C \\
388 A\end{array}$ \\
\hline & $\mathrm{CO}_{2}$ & $\begin{array}{l}\text { 7830G } \\
7918 \mathrm{~T}-7966 \mathrm{~T}-8117 \mathrm{~T} \\
7982 \mathrm{C} \\
7982 \mathrm{C}-8019 \mathrm{~T} \\
\text { 7985T } \\
7986 \mathrm{G}\end{array}$ \\
\hline & ND1 & $\begin{array}{l}3573 A-3632 A-3670 A \\
3905 G \\
3993 C\end{array}$ \\
\hline \multirow{3}{*}{ Non-AZT Treated } & HV2 & $\begin{array}{l}79 C \\
181 A \\
186 A-253 A-296 C \\
238 A \\
295 A \\
320 A-345 A \\
331 A \\
345 A \\
388 A\end{array}$ \\
\hline & $\mathrm{CO}_{2}$ & $\begin{array}{l}7718 \mathrm{~A} \\
7931 \mathrm{C}-7982 \mathrm{C} \\
7985 \mathrm{~T} \\
8087 \mathrm{G} \\
8206 \mathrm{~T}\end{array}$ \\
\hline & ND1 & $\begin{array}{l}3847 \mathrm{G} \\
3941 \mathrm{~A} \\
3947 \mathrm{~A} \\
4001 \mathrm{~T}\end{array}$ \\
\hline
\end{tabular}

Each line is a different sample. Instances of more than one mutation in a sequence are indicated by a hyphen between mutations. 
a statistically significant increase in mtDNA mutations in vitro in both primary and cancer cell lines of different cell types. The fluorescent signature shifts indicative of mutations, as proven by previous publications $[29,37,38]$ and confirmed here in multiple instances by sequencing, were not likely to be the result of amplification errors and were not the result of cloning errors [28]. While the total number of mutations after thirty days of treatment was relatively large, the number of mutations in individual experiments was small (Supplemental Table 2). Per thirty day experiment an average of 33, 24, and 40 and 34, 34, and 26 were seen in the HV2, CO2, and ND1 targets of HepG2 cells and CCD-1112Sk cells, respectively. These relatively low numbers of mutations in cultured cells are likely to be under estimates for several compounding technical reasons. First, the Light-On/Lights-Off method underestimates the frequency of sequence changes in mitochondrial DNA, because only those signatures shifts that lay outside three standard deviations of the reference for two consecutive temperature points are scored positive. In addition, some fluorescent signatures are due to more than one sequence change as revealed by sequencing (Table 3). Finally, the fact that each well had 1-5 molecules that are analysed as a single fluorescent signature shift also lowers the number of mutations recorded.

Several mechanisms may account for AZT-induced mtDNA mutation. The fact that there is no obvious pattern to the observed mutations (Table 3) suggests that AZT does not mutagenize mtDNA by direct incorporation. Alternatively, AZT could damage mtDNA by increasing ROS or damaging proteins, or by interacting with the mitochondrial DNA polymerase [18,39]. These hypotheses would account for the fact that AZT increases mtDNA mutations in the relatively short period of time observed in this study

This report is the first time that the Lights-On/Lights-Off method has been specifically used for mutation scanning at the digital or near digital level. The method is applicable to a wide variety of situations, such as aging and disease pathogenesis, or to test other drugs that are known to damage mitochondria, or have been correlated with chronic disease syndromes which are likely to be associated with random mutations accumulating in a target sequence. In these situations it is not necessary to know in advance if the target of interest has a "wild type" sequence, since the fluorescent signatures of new variants analysed at the neardigital level are compared to a fluorescent reference signature generated from large populations of starting genomes in which the contributions of rare variants are average out. The new method is useful because it is rapid, multiplexed, and minimizes the number of samples that actually need to be sequenced.

\section{Acknowledgements}

The authors would like to thank Dr. Reis for stimulating discussions about the assay and data analysis. This work was funded in part by Smiths Detection Diagnostics, Inc. to L.J.W.

Smiths Detection Diagnostics, Inc. had no role in study design; in the collection, analysis, and interpretation of data; in the writing of the report; and in the decision to submit the paper for publication.

\section{References}

1. Wallace DC (2010) Mitochondrial DNA mutations in disease and aging. Environ Mol Mutagen 51: 440-450.

2. Wallace DC, Fan W (2010) Energetics, epigenetics, mitochondrial genetics. Mitochondrion 10: 12-31.

3. Wallace DC (1994) Mitochondrial DNA sequence variation in human evolution and disease. Proc Natl Acad Sci U S A 91: 8739-8746.

4. Keane PC, Kurzawa M, Blain PG, Morris CM (2011) Mitochondrial dysfunction in Parkinson's disease. Parkinsons Dis 2011: 716871.
5. Swerdlow RH, Khan SM (2004) A "mitochondrial cascade hypothesis" for sporadic Alzheimer's disease. Med Hypotheses 63: 8-20.

6. Du H, Guo L, Yan S, Sosunov AA, McKhann GM, et al. (2010) Early deficits in synaptic mitochondria in an Alzheimer's disease mouse model. Proc Natl Acad Sci U S A 107: 18670-18675.

7. Pravenec M, Hyakukoku M, Houstek J, Zidek V, Landa V, et al. (2007) Direct linkage of mitochondrial genome variation to risk factors for type 2 diabetes in conplastic strains. Genome Res 17: 1319-1326.

8. Chen JZ, Kadlubar FF (2004) Mitochondrial mutagenesis and oxidative stress in human prostate cancer. J Environ Sci Health C Environ Carcinog Ecotoxicol Rev 22: 1-12.

9. Santos C, Martínez M, Lima M, Hao YJ, Simões N, et al. (2008) Mitochondrial DNA mutations in cancer: a review. Curr Top Med Chem 8: 1351-1366.

10. Koczor CA, Lewis W (2010) Nucleoside reverse transcriptase inhibitor toxicity and mitochondrial DNA. Expert Opin Drug Metab Toxicol 6: 1493-1504.

11. Senior K (2005) Growing old with HIV. Lancet Infect Dis 5: 739.

12. Lewis W, Gonzalez B, Chomyn A, Papoian T (1992) Zidovudine induces molecular, biochemical, and ultrastructural changes in rat skeletal muscle mitochondria. J Clin Invest 89: 1354-1360.

13. de Martino M, Zammarchi E, Filippi L, Donati MA, Mannelli F, et al. (1995) Redox potential status in children with perinatal HIV-1 infection treated with zidovudine. AIDS 9: 1381-1383.

14. Dykens JA, Will $Y(2007)$ The significance of mitochondrial toxicity testing in drug development. Drug Discov Today 12: 777-785.

15. Kohler JJ, Lewis W (2007) A brief overview of mechanisms of mitochondrial toxicity from NRTIs. Environ Mol Mutagen 48: 166-172.

16. Hanes JW, Johnson KA (2007) A novel mechanism of selectivity against AZT by the human mitochondrial DNA polymerase. Nucleic Acids Res 35: 6973-6983.

17. Scruggs ER, Dirks Naylor AJ (2008) Mechanisms of zidovudine-induced mitochondrial toxicity and myopathy. Pharmacology $82: 83-88$.

18. Wendelsdorf KV, Song Z, Cao Y, Samuels DC (2009) An analysis of enzyme kinetics data for mitochondrial DNA strand termination by nucleoside reverse transcription inhibitors. PLoS Comput Biol 5: e1000261.

19. Pan-Zhou XR, Cui L, Zhou XJ, Sommadossi JP, Darley-Usmar VM (2000) Differential effects of antiretroviral nucleoside analogs on mitochondrial function in HepG2 cells. Antimicrob Agents Chemother 44: 496-503.

20. Höschele D (2006) Cell culture models for the investigation of NRTI-induced mitochondrial toxicity. Relevance for the prediction of clinical toxicity. Toxicol In Vitro 20: 535-546.

21. Pereira CV, Moreira AC, Pereira SP, Machado NG, Carvalho FS, et al. (2009) Investigating drug-induced mitochondrial toxicity: a biosensor to increase drug safety? Curr Drug Saf 4: 34-54.

22. Lane N (2011) Mitonuclear match: optimizing fitness and fertility over generations drives ageing within generations. Bioessays 33: 860-869.

23. Onyango I, Khan S, Miller B, Swerdlow R, Trimmer P, et al. (2006) Mitochondria genomic contribution to mitochondrial dysfunction in Alzheimer's disease. J Alzheimers Dis 9: 183-193.

24. Holt IJ (2010) Zen and the art of mitochondrial DNA maintenance. Trends Genet 26: 103-109.

25. Martin AM, Hammond E, Nolan D, Pace C, Den Boer M, et al. (2003) Accumulation of mitochondrial DNA mutations in human immunodeficiency virus-infected patients treated with nucleoside-analogue reverse-transcriptase inhibitors. Am J Hum Genet 72: 549-560.

26. Payne BA, Wilson IJ, Hateley CA, Horvath R, Santibanez-Koref M, et al. (2011) Mitochondrial aging is accelerated by anti-retroviral therapy through the clonal expansion of mtDNA mutations. Nat Genet 43: 806-810.

27. Walker UA, Setzer B, Venhoff N (2002) Increased long-term mitochondria toxicity in combinations of nucleoside analogue reverse-transcriptase inhibitors AIDS 16: 2165-2173.

28. Osborne A, Reis AH, Bach L, Wangh LJ (2009) Single-molecule LATE-PCR analysis of human mitochondrial genomic sequence variations. PLoS One 4: e5636. 
Citation: Osborne AE, Rice JE, Sanchez AJ, Wangh LJ (2013) AZT Treatment Increases mtDNA Mutations in HepG2 and CCD-1112Sk Cells. J AIDS Clin Res 4: 250. doi: 10.4172/2155-6113.1000250

29. Rice JE, Reis AH Jr, Rice LM, Carver-Brown RK, Wangh LJ (2012) Fluorescent signatures for variable DNA sequences. Nucleic Acids Res 40: e164.

30. Marroquin LD, Hynes J, Dykens JA, Jamieson JD, Will Y (2007) Circumventing the Crabtree effect: replacing media glucose with galactose increases susceptibility of HepG2 cells to mitochondrial toxicants. Toxicol Sci 97: 539547

31. Pierce KE, Rice JE, Sanchez JA, Wangh LJ (2002) QuantiLyse: reliable DNA amplification from single cells. Biotechniques 32: 1106-1111.

32. Kent WJ, Sugnet CW, Furey TS, Roskin KM, Pringle TH, et al. (2002) The human genome browser at UCSC. Genome Res 12: 996-1006.

33. Rice JE, Sanchez JA, Pierce KE, Reis AH Jr, Osborne A, et al. (2007) Monoplex/ multiplex linear-after-the-exponential-PCR assays combined with PrimeSafe and Dilute-'N'-Go sequencing. Nat Protoc 2: 2429-2438.

34. Jia Y, Osborne A, Rice JE, Wangh LJ (2010) Dilute-'N'-Go dideoxy sequencing of all DNA strands generated in multiplex LATE-PCR assays. Nucleic Acids Res 38: e119.
35. Côté HC, Brumme ZL, Craib KJ, Alexander CS, Wynhoven B, et al. (2002) Changes in mitochondrial DNA as a marker of nucleoside toxicity in HIVinfected patients. N Engl J Med 346: 811-820.

36. Garrabou G, López S, Morén C, Martínez E, Fontdevila J, et al. (2011) Mitochondrial damage in adipose tissue of untreated HIV-infected patients. AIDS 25: 165-170.

37. Pierce KE, Peter H, Bachmann TT, Volpe C, Mistry R, et al. (2013) Rapid detection of TEM-type extended-spectrum $\hat{i}^{2}$-lactamase (ESBL) mutations using lights-on/lights-off probes with single-stranded DNA amplification. J Mol Diagn 15: 291-298.

38. Carver-Brown RK, Reis AH Jr, Rice LM, Czajka JW, Wangh LJ (2012) Design and Construction of a Single-Tube, LATE-PCR, Multiplex Endpoint Assay with Lights-On/Lights-Off Probes for the Detection of Pathogens Associated with Sepsis. J Pathog 2012: 424808.

39. Lim SE, Copeland WC (2001) Differential incorporation and removal of antivira deoxynucleotides by human DNA polymerase gamma. J Biol Chem 276: 23616-23623. 